\title{
PROGNOSTIC FACTORS IN THE SURVIVAL OF PATIENTS DIAGNOSED WITH PRIMARY NON- METASTATIC OSTEOSARCOMA WITH A POOR RESPONSE TO NEOADJUVANT CHEMOTHERAPY
}

\author{
Rosalvo Zósimo Bispo Júnior, ${ }^{\mathrm{I}}$ Olavo Pires de Camargo ${ }^{\mathrm{II}}$
}

doi: $10.1590 / \mathbf{S 1 8 0 7 - 5 9 3 2 2 0 0 9 0 0 1 2 0 0 0 0 7}$

\begin{abstract}
Bispo Júnior RZ, Camargo OP. Prognostic factors in the survival of patients diagnosed with primary non-metastatic osteosarcoma with a poor response to neoadjuvant chemotherapy. Clinics. 2009;64(12):1177-86.
\end{abstract}

INTRODUCTION: Identification of variables that determine the prognosis for osteosarcoma may enable stratification of patients into subgroups with better or worse risk of local recurrence, metastases and death due to the disease. Discovery of such prognostic factors would permit selection of a subgroup of at-risk patients, with the aim of improving the therapeutic effectiveness.

OBJECTIVE: To identify prognostic factors related to local recurrence-free survival, metastasis-free survival and overall survival among patients with highly malignant primary osteosarcoma that was non-metastatic on diagnosis and had poor response to neoadjuvant chemotherapy.

SAMPLE AND METHODS: Out of 45 patients admitted to a referral center in Brazil between 2000 and 2004,24 were selected for this study.

RESULTS: The adverse factors that influenced the risk of local recurrence and the overall survival in univariate analysis were histological subtype other than osteoblastic $(\mathrm{p}=0.017)$ and tumor size greater than $15 \mathrm{~cm}(\mathrm{p}=0.048)$. In relation to metastasis-free survival, the non-osteoblastic subtype had a worse prognosis $(\mathrm{p}=0.007)$. The association of histological subtype maintained its significance in multivariate analysis for all studied survival categories.

CONCLUSIONS: Tumor size greater than $15 \mathrm{~cm}$ is an adverse factor for local recurrence-free survival and overall survival but did not influence metastasis-free survival. The osteosarcoma histological type is a significant independent predictor for local recurrencefree survival, metastasis-free survival and overall survival.

KEYWORDS: Bone neoplasms; Pathology; Epidemiology; Surgery; Drug therapy.

\section{INTRODUCTION}

Boyer (1805) apud Mehlman \& Cripe (2008) ${ }^{1}$ was the first to use the term "osteosarcoma", referring to a distinct entity apart from more frequent bone lesions. Primary tumors affecting bone tissue are comparatively rare among neoplasias. $^{2}$

\footnotetext{
I Faculty of Medicine, University of Campina Grande - Campina Grande/ PB, Brazil.

II Department of Orthopedics and Traumatology, Faculdade de Medicina da Universidade de São Paulo - São Paulo/SP, Brazil.

Email: zosimo_jr@yahoo.com.br

Tel: 55832106.9235

Received for publication on July 08,2009

Accepted for publication on September 17, 2009
}

Osteosarcomas may be primary or secondary. The latter are sarcoma forms that occur in bones affected by preexisting abnormalities, ${ }^{3}$ such as Paget's disease, radiation lesions, bone infarct, fibrous dysplasia or osteomyelitis. ${ }^{3-5}$ Among primary osteosarcomas, these precursor conditions are not observed.

Primary osteosarcomas are considered to be a disease of young people, ${ }^{6}$ with peak incidence in the second decade of life. ${ }^{4,5}$ Among older children and adolescents (12-18 years), they are the third most common type of neoplasia, preceded by leukemia and lymphoma. ${ }^{7}$ Their etiology remains unknown, ${ }^{4,6}$ but it is known that the period of their greatest incidence coincides with the growth spurt of puberty. This period is characterized by rapid growth of the long bones, suggesting a correlation between this phase 
of life and appearance of these tumors. ${ }^{5,8}$ They may occur in any anatomical location. They preferentially affect the metaphyses of long bones; the three main sites are the distal femur, tibia and proximal humerus. , $^{6-15}$

Histologically, osteosarcomas can be classified into several types: osteoblastic, chondroblastic, fibroblastic, rich in giant cells, epithelioid, small cells and telangiectasic, depending on the dominant element. The locations of these lesions in the bone are named parosteal, periosteal and intramedullary. The conventional type, emerging from the intramedullary cavity, represents around $75 \%$ of all osteosarcomas. ${ }^{16}$

Clinically, these tumors are initially manifested as local pain that is generally nonspecific, with or without an associated palpable tumor. This presentation may occur over periods ranging from weeks to months. ${ }^{4-6,17}$

Over recent decades, there have been major changes in the diagnosis and treatment of these tumors, giving rise to significant increases in five-year survival among such patients. Formerly, the five-year survival rate was approximately $10-25 \%$, but it is now around $50-75 \%$, according to American and European studies. ${ }^{7,11,12,18-25}$ Brazilian studies ${ }^{10,15,26}$ have also demonstrated this evolution in multidisciplinary therapy, although not reaching the rates cited in other countries.

Identification of variables that determine the prognosis for osteosarcoma may permit stratification of these patients into subgroups with better or worse risk of local recurrence, metastasis and death due to this disease. Discovery of such prognostic factors would allow at-risk patients to be selected with the aim of improving the therapeutic results.

The aim of the present study, which is part of a general investigation on sarcomas, ${ }^{27,28}$ was to identify prognostic factors relating to local recurrence-free survival (LRFS), metastasis-free survival (MFS) and overall survival (OS) among patients with highly malignant primary osteosarcoma that was non-metastatic on diagnosis, with a poor response to neoadjuvant chemotherapy.

\section{SAMPLE AND METHODS}

\section{Inclusion criteria}

All of the patients included in the present study fulfilled the following eligibility criteria: (a) there was anatomopathological confirmation of primary osteosarcoma following review of all of the slides by a pathologist specializing in musculoskeletal tissues; (b) the patients were treated (during this period) with the same neoadjuvant chemotherapy protocol (GBTO Study V); (c) the patients were operated on at our center to achieve local control over the primary tumor following chemotherapy; and (d) the patients were considered to present poor responses to the preoperative chemotherapy (grades I and II), according to the classification of HUVOS et al. ${ }^{29}$ (1977).

The medical files of 45 patients with an anatomopathological diagnosis of primary osteosarcoma made by the Orthopedic Oncology Group at a referral center in Brazil between 2000 and 2004 were evaluated. These patients had been treated in accordance with the recommendations from Study V of the Brazilian Cooperative Group for Osteosarcoma Treatment (GBTO). Among these 45 patients, 19 were excluded from the study because, at the time of diagnosis, they presented metastases at the initial evaluation. Another two cases were also excluded: one patient, with a low degree of malignancy, did not undergo neoadjuvant chemotherapy (surface osteosarcoma; parosteal); the other patient died before the operation, due to complications from the chemotherapy. Thus, the variables in the medical files of 24 patients who underwent treatment at our center were analyzed. This project was approved by the Research Ethics Committee of the participating institution.

\section{Casuistic analysis}

\section{Gender and age}

Among the 24 patients studied, 14 (58.3\%) were male and $10(41.7 \%)$ were female, with a proportion of 1.4:1.0. Their ages ranged from 5 to 44 years, with a mean of 15.75 \pm 7.57 years (median of 15 years).

\section{Anatomical location}

Seventeen $(70.8 \%)$ of the patients presented with a tumor in the femur, particularly in its distal third (Figure 1). Among
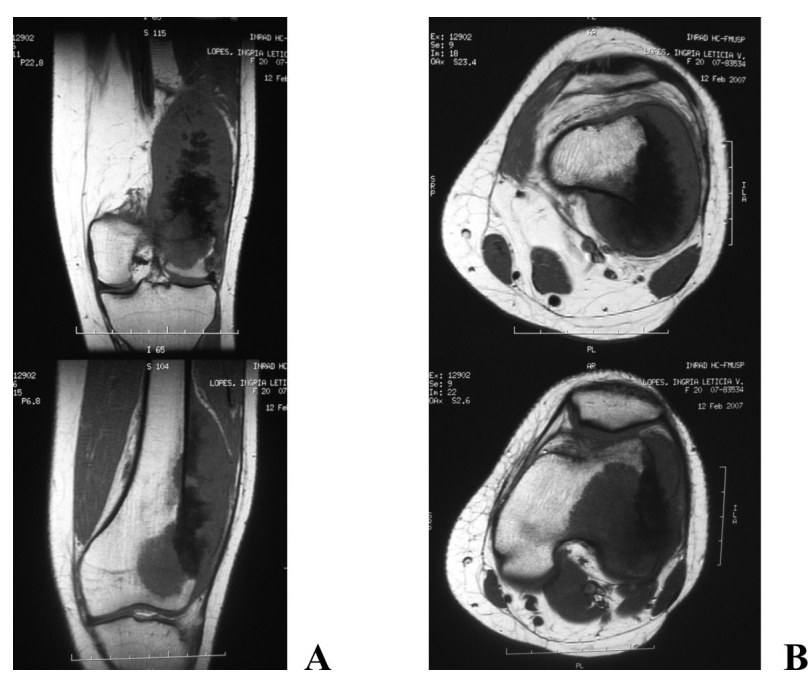

Figure 1 - Magnetic resonance images demonstrating the tumor expansion process (osteosarcoma) in the distal metaphysis and epiphysis of the left femur and affecting soft tissues (extra-compartmental). Coronal (A) and axial (B) T1 images 
the remaining patients, the tibia was affected in four cases $(16.7 \%)$ and the humerus in two $(8.3 \%)$, all in the proximal region. The maxilla was affected in one case $(4.2 \%)$. The knee region (distal femur and proximal tibia) was the most frequent location, accounting for $87.5 \%$ of the cases.

\section{Clinical condition and its duration}

All of the patients presented clinical complaints of local pain, except for the cases of pathological fracture $(12.5 \%)$. The tumor was the most frequent sign. It occurred in association with pain in $58.3 \%$ of the cases. The time between sign or symptom onset and seeking of medical care ranged from 0 to 12 months, with a mean of $3.67+/-2.81$ months (median of three months) among the patients studied.

\section{Assessment criteria of variables}

\section{Epidemiological and clinical variables}

Gender - male or female;

Age - less than or equal to 15 and greater than 15 years old (median);

Anatomical location - local presentation was divided into the femur or extra-femur;

Clinical condition - two groups were formed: with or without pathologic fracture;

Delay of clinical condition - less than to 3 and greater than or equal to three months (median).

\section{Anatomopathological variables}

Histological type - osteoblastic or non-osteoblastic variants; Tumor size - less than or equal to 15 and greater than 15 $\mathrm{cm} ;{ }^{10}$

Surgical margins - less than or equal to 2 and greater than $2 \mathrm{~mm}$ (mean).

\section{Therapeutic variables}

Surgical treatment - tumor resection was wide or radical. ${ }^{30}$

\section{Statistical analysis}

Relevant medical data was extracted from medical files, using a defined protocol, and assembled in a database (Excel 2007, Windows software). Statistical analyses were performed using SPSS statistical software (Version 12.0; SPSS Inc., Chicago, IL, USA).

Descriptive analysis was performed using means, medians and percentages. The simple frequencies of all of the studied variables were calculated. The accumulated survival chances were determined using the Kaplan-Meier technique. All of the variables were analyzed with regard to their importance for the prognoses of LRFS, MFS and OS.
We simplified the numbers by means of standardized rounding techniques, with elimination of the decimal places for the frequencies and survival rates. Multivariate analysis was performed using the logistic regression technique, with the Cox proportional hazards model. Statistical significance was defined as $\mathrm{p}<0.05$.

\section{RESULTS}

\section{Epidemiological and clinical variables}

\section{Pulmonary metastasis}

Pulmonary metastasis was identified in nine patients $(37 \%)$. All of them developed pulmonary metastasis after they had been diagnosed with osteosarcoma. Eight progressed to death after a follow-up ranging from 2 to 54 months. The eighth patient was still alive with pulmonary disease at 17 months after the diagnosis.

\section{Length of follow-up}

The mean length of follow-up among the patients with non-metastatic osteosarcoma in our sample was 39.08 months (with a standard deviation of 28.16 months and median of 47 months), ranging from 2 to 83 months.

\section{Death due to neoplasia}

The death rate consequent to osteosarcoma was $41.7 \%$ (10 cases).

\section{Anatomopathological variables}

\section{Histology and tumor size}

The histological type was reviewed by a pathologist after surgical resection. The osteoblastic variant was the most common histological type, affecting 10 of the 24 cases. The case frequencies according to histological subtype of osteosarcoma were osteoblastic $(41.7 \%)$ and nonosteoblastic $(58.3 \%)$ (chondroblastic, mixed, telangiectasic, round-cell rich and fibroblastic) (Table 1) (Figure 2). The tumor size ranged from 4 to $29 \mathrm{~cm}$ along its greatest dimension, with a mean of $12.26+/-6.15 \mathrm{~cm}$ (median of $10.50 \mathrm{~cm}$ ), according to measurements of the specimens after surgical resection. Subsequently, the tumors were divided into two groups, according to PETRILLI et al. ${ }^{10}$ (1991): less than or equal to $15 \mathrm{~cm}(78.3 \%)$ and greater than $15 \mathrm{~cm}(21.7 \%)$ (Table 1).

\section{Surgical margins}

The width of the free margin of the surgical resection (type wide-limb salvage) ranged from 0.10 to $1.00 \mathrm{~cm}$, with a mean of $0.26 \mathrm{~cm}$ (standard deviation of 0.24 ) and median 


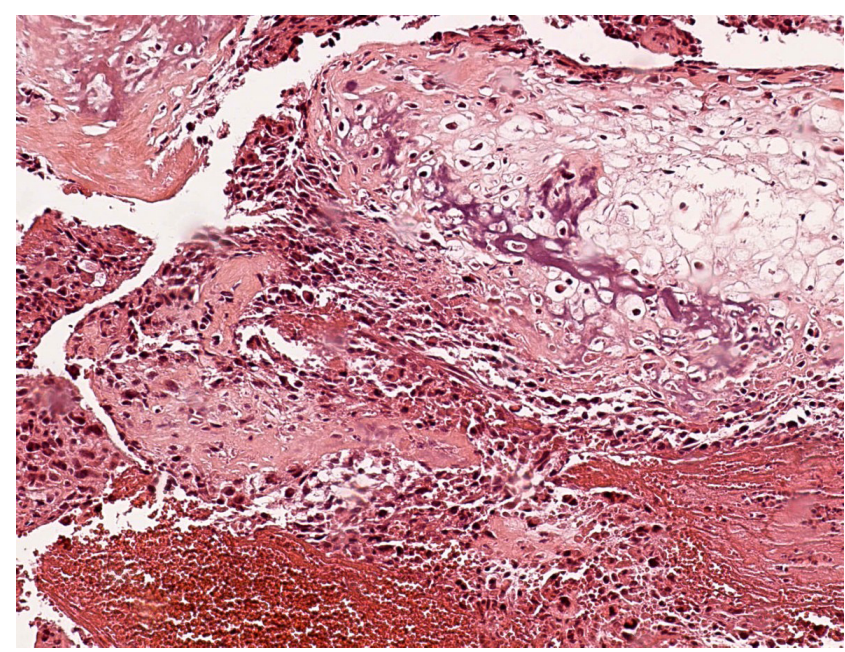

Figure 2 - Photomicrograph showing the telangiectasia type of osteosarcoma (non-osteoblastic) (200 X- HE)

Table 1 - Distribution of select anatomopathological variables for the 24 patients with primary osteosarcoma that was nonmetastatic at diagnosis

\begin{tabular}{llcc}
\hline Variable & Category & $\begin{array}{c}\text { Frequency } \\
(\boldsymbol{n})\end{array}$ & Percent $(\%)$ \\
\hline Histological Type & Osteoblastic (O) & 10 & 41.7 \\
& Chondroblastic (C) & 5 & 20.8 \\
& Fibroblastic & 1 & 4.2 \\
& Mixed (O+C) & 3 & 12.5 \\
& Round-cell rich & 1 & 4.2 \\
& Telangiectasic & 2 & 8.3 \\
& Not classifiable & 2 & 8.3 \\
Tumor Size* $(\mathrm{cm})$ & $\leq 15$ & 18 & 78.3 \\
& $>15$ & 5 & 21.7 \\
\hline
\end{tabular}

* Data available for 23 patients.

of $0.10 \mathrm{~cm}$. In radical resection (ablative), the margins were greater.

\section{Therapeutic variables}

\section{Surgical and adjuvant treatment}

In all cases, the tumor resection was wide (22 cases) or radical ( 2 cases), according to the classification of ENNEKING et al. ${ }^{30}$ (1980). The surgery preserved limbs and limb function in the majority of the cases (91.7\%) (Figure 3 ). The bone material was replaced by unconventional endoprostheses in $81.8 \%$ of the cases and by allografts in $18.2 \%$ (Figure 4). All of the patients received preoperative chemotherapy in accordance with the recommendations from GBTO Study V [with cisplatin $\left(120 \mathrm{mg} / \mathrm{m}^{2} /\right.$ cycle $)$, ifosfamide $\left(13.5 \mathrm{~g} / \mathrm{m}^{2} /\right.$ cycle $)$ and adriamicin $\left(80 \mathrm{mg} / \mathrm{m}^{2} /\right.$

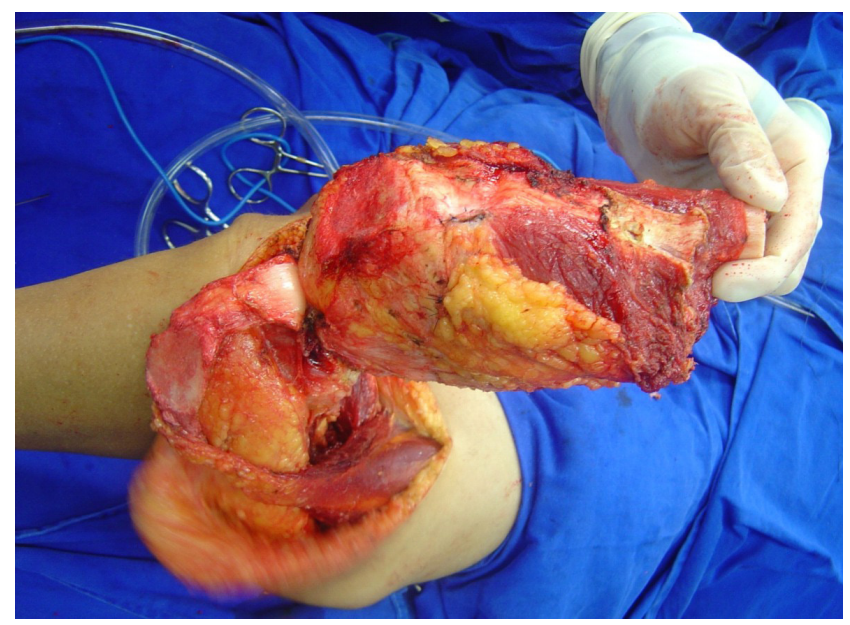

Figure 3 - Intraoperative photograph showing a wide tumor resection in the distal region of the left femur

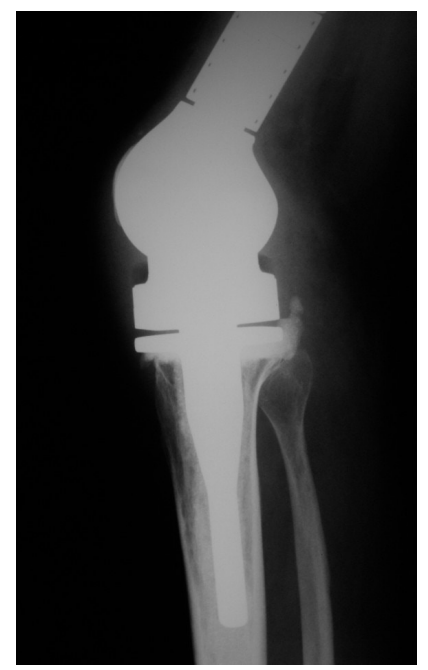

Figure 4 - Radiograph showing a lateral view of the knee region, in which osteoarticular substitution using an unconventional modular endoprosthesis can be seen

cycle), totaling nine cycles for patients who were nonmetastatic at diagnosis].

\section{Local recurrence}

Local recurrence was observed in two cases (8.3\%), both of which were non-osteoblastic. These two patients (100\%) with local recurrence presented pulmonary metastases and progressed to death.

\section{Prognostic factors relating to local recurrence-free survival}

The LRFS curve for the 24 patients is presented in Figure 5. The LRFS rates for these patients were $61 \%$ and $55 \%$, respectively, for two and five years. The factors that influenced the risk of local recurrence in the univariate analysis were tumor size $(\mathrm{p}=0.048)$ and histological 


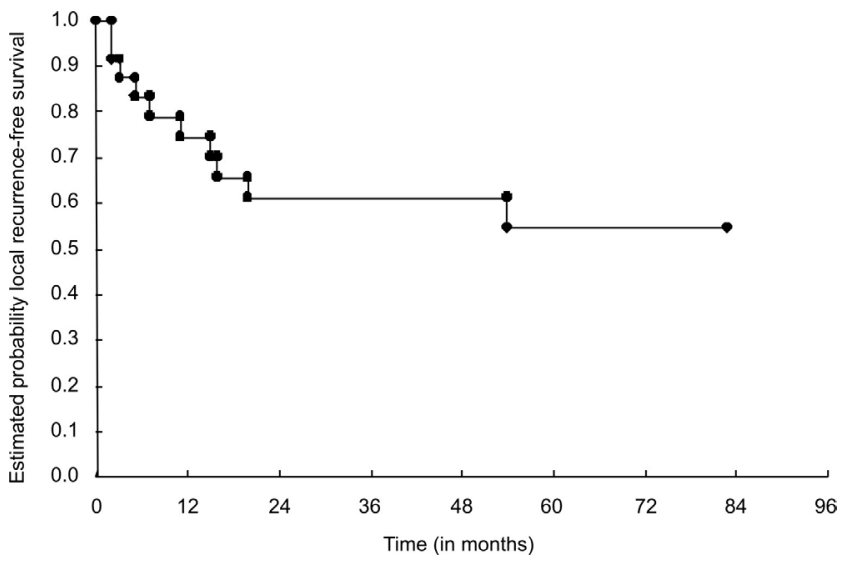

Figure 5 - Curve of local recurrence-free survival in months for the 24 patients with primary osteosarcoma that was non-metastatic at diagnosis

subtype $(\mathrm{p}=0.017)$. The presence of a tumor measuring more than $15 \mathrm{~cm}$ along its greatest axis was considered to be an adverse factor. Subtypes that were considered to be non-osteoblastic presented a greater local recurrence rate than did the osteoblastic types. The survival curves for the variables with statistical significance (histological type and tumor size) are presented in Figures 6 and 7.

The other studied factors did not reach statistical significance (gender, age, tumor location, clinical condition and duration, surgical margins and type of surgical treatment).

Multivariate analysis was performed using the Cox proportional hazards logistic regression model to evaluate the risk of local recurrence. For this analysis, the variables of histological type and tumor size were used, since these variables reached $\mathrm{p}<0.10$ in the univariate analysis (KaplanMeier curve). We applied a stepwise variable selection

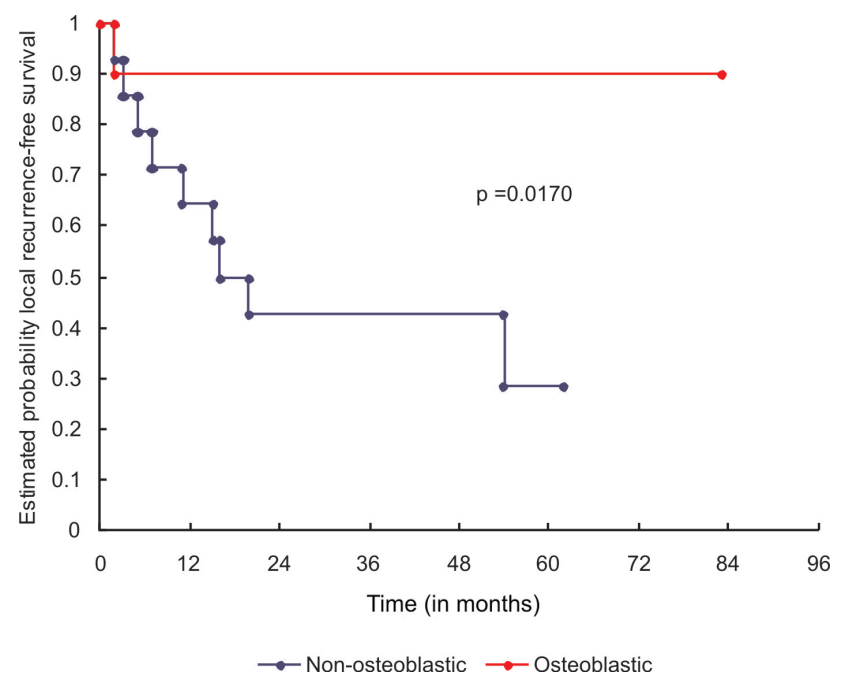

Figure 6 - Curve of local recurrence-free survival in months for the 24 patients with primary osteosarcoma that was non-metastatic at diagnosis, according to histological type

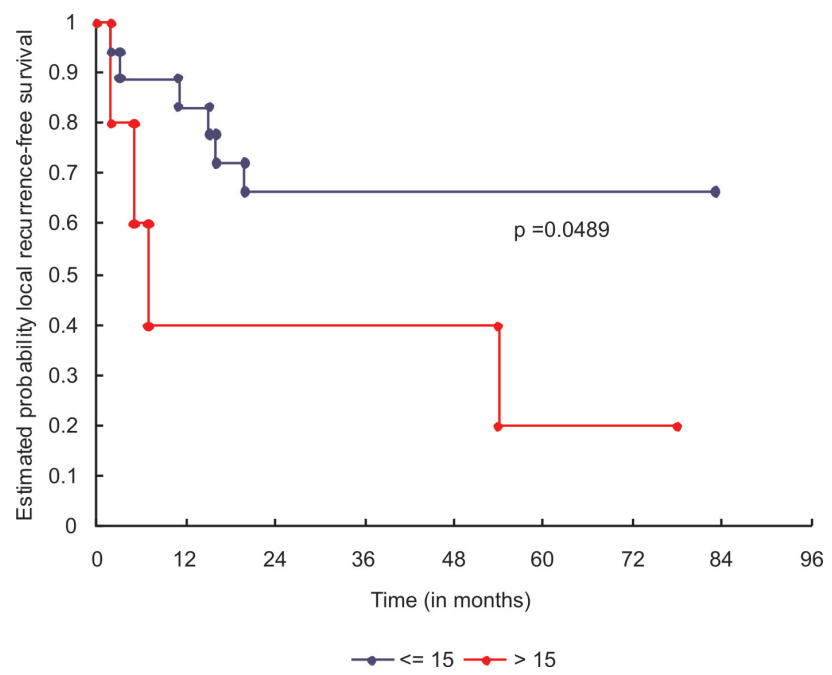

Figure 7 - Curve of local recurrence-free survival in months for the 23 patients with primary osteosarcoma that was non-metastatic at diagnosis, according to tumor size

process to the model and observed that histological type was associated with LRFS. Thus, the risk that a patient with local recurrence would not survive was 8.4 times greater when the histological type was non-osteoblastic.

\section{Prognostic factors relating to metastasis-free survival}

The MFS curve for the 24 patients is presented in Figure 8. The MFS rates for these patients were $57 \%$ and $51 \%$ for two and five years, respectively. Histological subtype was the only factor that influenced the risk of metastasis in the univariate analysis $(\mathrm{p}=0.007)$. The non-osteoblastic subtype presented a higher rate of metastasis than did the osteoblastic subtype. The survival curve for the variable presenting statistical significance (histological type) is shown in Figure 9.

The other factors did not reach statistical significance (age, gender, tumor location, clinical condition and duration, tumor size, surgical margins and type of surgical treatment).

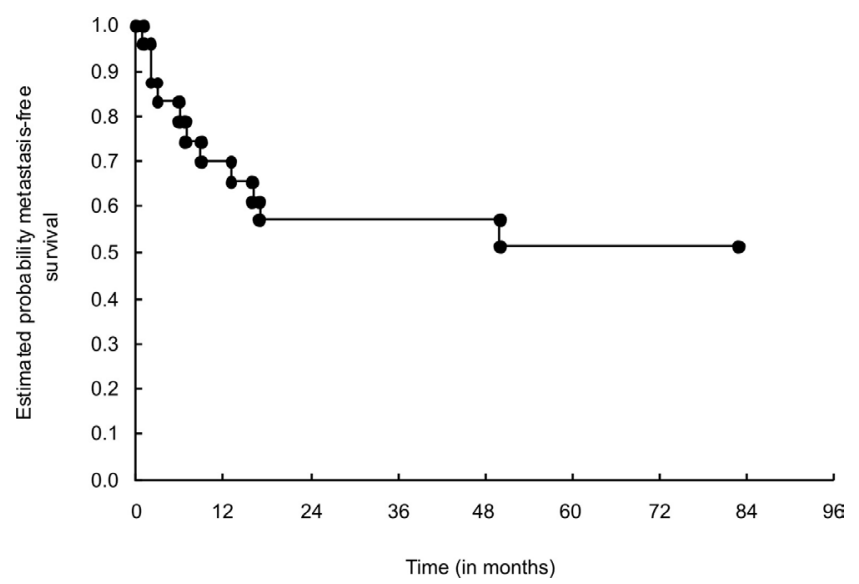

Figure 8 - Curve of metastasis-free survival in months for the 24 patients with primary osteosarcoma that was non-metastatic at diagnosis 


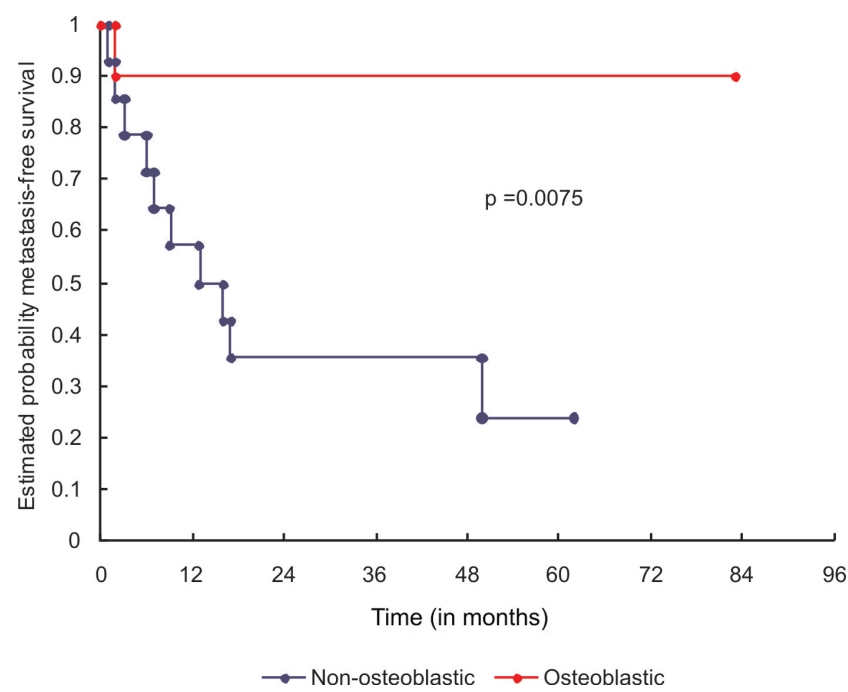

Figure 9 - Curve of metastasis-free survival in months for the 24 patients with primary osteosarcoma that was non-metastatic at diagnosis, according to histological type

We observed that the parameter of histological type was the only factor that presented statistical significance in the multivariate analysis using the Cox regression model for MFS. Thus, the risk that a patient presenting pulmonary metastasis over the course of the treatment would not survive was 10 times greater when the histological type presented was non-osteoblastic.

\section{Prognostic factors relating to overall survival}

The OS rates for the 24 patients studied were $61 \%$ and $55 \%$ for two and five years, respectively (Figure 10). The factors that influenced the risk of death in the univariate analysis were histological subtype $(\mathrm{p}=0.017)$ and tumor size $(p=0.048)$. The survival curves for the variables presenting statistical significance (tumor size and histological type) are shown in Figures 11 and 12.

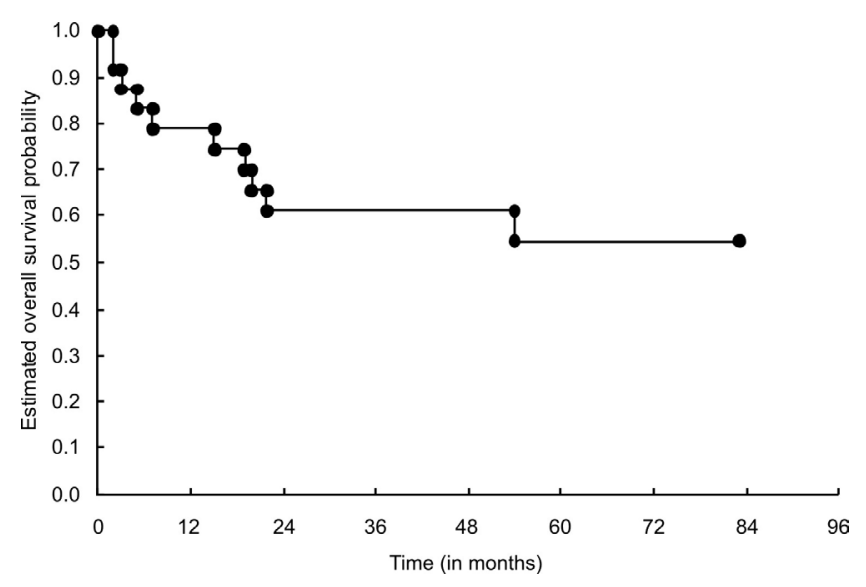

Figure 10 - Curve of overall survival in months for the 24 patients with primary osteosarcoma that was non-metastatic at diagnosis

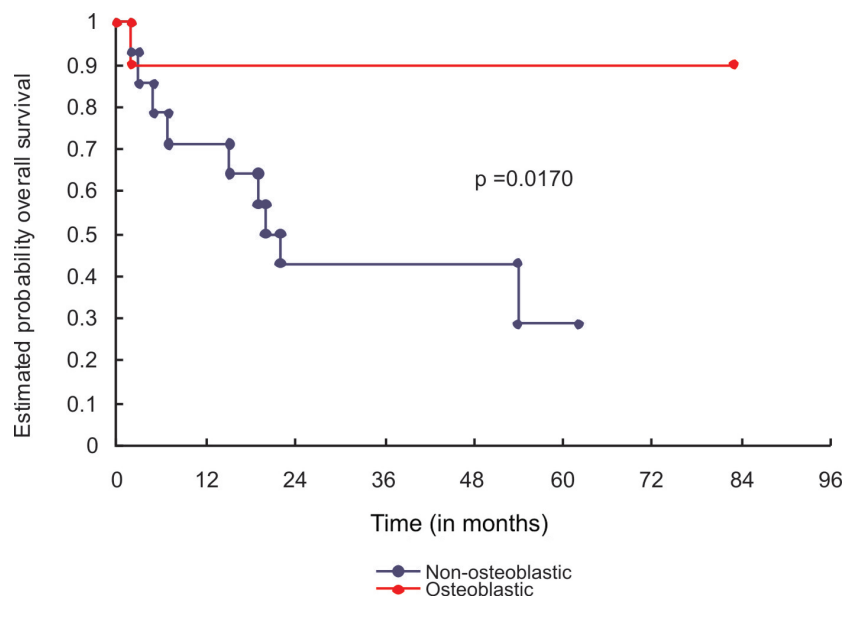

Figure 11 - Curve of overall survival in months for the 24 patients with primary osteosarcoma that was non-metastatic at diagnosis, according to histological type

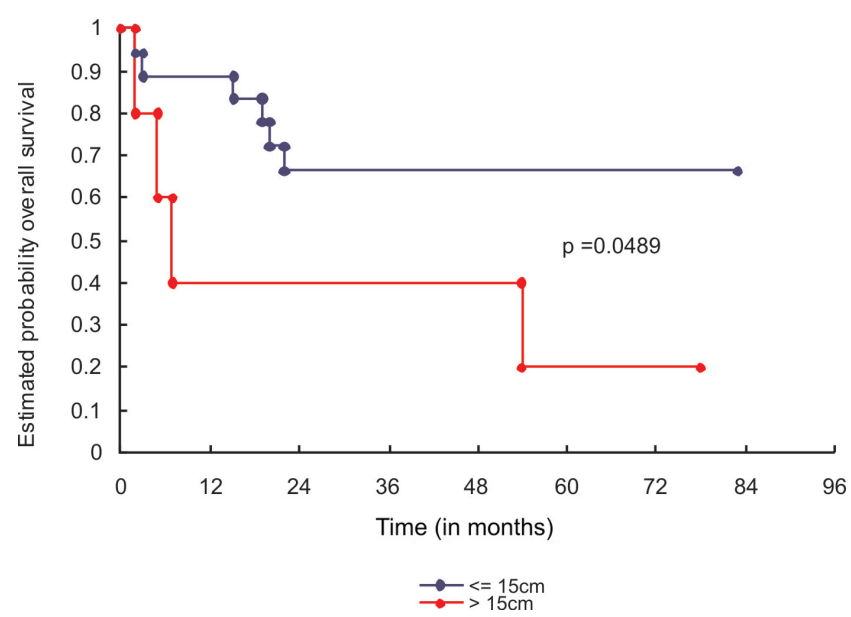

Figure 12 - Curve of overall survival in months for the 23 patients with primary osteosarcoma that was non-metastatic at diagnosis, according to tumor size

Gender, age, tumor location, clinical condition and duration, surgical margins and type of surgical treatment did not reach the statistically significant level $(\mathrm{p}<0.05)$.

For the multivariate analysis, we used the variables of histological type and tumor size (which presented with $\mathrm{p}$ $<0.10$ in the univariate analysis). We applied a stepwise variable selection process to the model and observed that histological type was associated with OS. The risk that a patient would not survive was 8.4 times greater when the histological type was non-osteoblastic.

\section{DISCUSSION}

Several clinical studies ${ }^{10,11,13-15,19,25,26,31-43}$ aiming to identify prognostic factors that might influence the survival of patients with osteosarcoma have been published. However, because of the great variation in the methodology 
used in these studies, the results are inconsistent and it is difficult to determine the real prognostic effects of the evaluated variables. ${ }^{31}$

The low frequency of this disease in itself would explain our small sample size. In addition, we used several rigid inclusion and exclusion criteria in this study with the aims of maintaining a homogeneous sample and, thus, faithfully evaluating the prognostic factors.

Overall, the age distribution of these tumors was bimodal. The first and larger peak of incidence was found to occur during the second decade of life. ${ }^{4,5}$ The second and smaller peak was seen among patients over 40-50 years of age, ${ }^{3,5}$ and has been found to be secondary to a preexisting bone abnormality. ${ }^{3-5}$ Classically, patients with primary osteosarcoma have been young and male,,$^{4,6,10-13,32}$ and the results from our series are concordant with this picture. The variables of age $e^{25,33,34}$ and gender ${ }^{33,35}$ have been shown to be prognostic factors in some series using univariate analysis alone. However, PETRILLI et al..$^{10}$ (1991) stated that male gender was a poor prognostic factor and FERRARI et al. ${ }^{13}$ (2001) indicated that age significantly influenced the survival of their patients in a double analysis (univariate and bivariate). In the present study, gender and age were not found to be significant prognostic variables, in agreement with other studies. ${ }^{19,36}$

With regard to the anatomical region most affected by these tumors, the literature shows that the femur is the most frequent site, followed by the tibia and humerus. ${ }^{6,9,10-15,17,32}$ In the present study, we also found that these sites were the most affected locations. In investigations of the tumor site as a prognostic variable, some studies have demonstrated that axial tumors have a relatively greater adverse effect than do those of the appendicular skeleton. ${ }^{25,37}$ However, our finding with the present series do not agree with those findings, most likely because we only had one axial case (affecting the maxilla). In relation to the bone, GLASSER et al. ${ }^{11}$ (1992) and PAKOS et al. ${ }^{32}$ (2009) reported that the prognosis for tumors located in the femur was worse. By contrast, GOORIN et al..$^{35}$ (1987) observed a worse prognosis for tumors in the humerus. Our data are not in agreement with this finding: we did not find that the location of the osteosarcoma was an adverse factor, and our result was similar to that of many other papers. ${ }^{12,19,22,38}$

Two literature reviews on prognostic factors for osteosarcoma $^{31,39}$ have stated that age, gender and tumor location were not as fully addressed in the literature as relevant variables.

Clinical complaints of pain are frequently encountered. This symptom is generally considered a warning, except in cases of pathological fracture. In the present series, complaints of locally increased volume were associated with painful processes in over half of the cases, similar to the finding of another Brazilian study. ${ }^{14}$

In a different series of osteosarcoma cases in the literature, ${ }^{11,17,19}$ the pathological fracture rate ranges from 7 to $17 \%$, which is compatible with the result of the present study (12.5\%). In agreement with some studies, ${ }^{11,19}$ we did not observe a worse prognosis for cases with a pathological fracture at the initial diagnosis among our samples.

Among our patients, the mean amount of time that elapsed between the first signs and symptoms and seeking medical care was approximately four months ranging from 0 to 12 months. Despite this large range, the length of time that had passed between the signs and symptoms did not demonstrate a worse prognosis with regard to the studied survival parameters. This association was also found in the series reported by PETRILLI et al. ${ }^{15}$ (2006). Other Brazilian authors ${ }^{15,17}$ have also shown higher means than that found in studies in other countries. ${ }^{25}$ These data suggest that the stage of the disease at the time of its initial presentation depends more on the biological properties of the tumor than on late diagnosis..$^{38}$ Nonetheless, it is important to emphasize that, in the present series, only patients who were non-metastatic at the time of diagnosis were included.

Adequate follow-up for these patients made it possible to identify pulmonary metastasis in about one-third by means of thoracic Computed Tomography. The presence of metastases is directly related to a worse prognosis regarding survival: eight of the nine patients who developed metastatic disease died after a period ranging from 2 to 54 months $(88.8 \%)$.

In the majority of studies, ${ }^{2,10,11,13,14}$ the osteoblastic variant was the most common histological type. The present study found the same result. According to some studies, the histological type is a strong predictor of LRFS, MFS and OS according to both univariate and multivariate analyses. ${ }^{10,13}$ The risk that patients with local recurrence would not survive was 8.4 times greater when they presented with non-osteoblastic tumors. Furthermore, the risk that patients with osteosarcoma who developed pulmonary metastases would not survive was 10 times greater when they presented with non-osteoblastic tumors. However, in disagreement with this finding, some papers, ${ }^{19,40,41}$ with stratification of the non-osteoblastic osteosarcoma (into types), demonstrated that the subtype differentially influenced prognosis. This association was especially true concerning the chondroblastic, telangiectasic or fibroblastic type, which had a more favorable prognosis than the osteoblastic type. It is interesting to note that patients who have a poor response to chemotherapy represent the present series.

Contradictory results are present in the literature, including in the principal reviews, regarding the correlation between tumor size and the prognosis for the patient. ${ }^{31,39}$ 
The parameters used in different studies (cutoff points) and the way of measuring tumor size (from one to three dimensions) may be the main reasons for the lack of consensus. ${ }^{10,12,13,15,19,25,31,32,39}$ In the present series, only onefifth of the resected osteosarcomas measured more than $15 \mathrm{~cm}$ along their longest axis. However, this minority presented worse results in relation to LRFS ( $p=0.048$ ) and OS $(p=0.048)$ according to the univariate analysis, thereby corroborating the data presented by several other studies. ${ }^{10,13,15,33,34,39,42}$ In our multivariate analysis, this prognostic variable (size) did not maintain significance. This finding differed from the studies of PETRILLI et al. ${ }^{10,15}$ (1991, 2006), despite the different cutoff points used in those two studies: 15 and $12 \mathrm{~cm}$, respectively.

Choosing between the type of surgical procedure (conservative or ablative) depends on several factors. Surgical treatment has now attained a limb preservation rate of around $50-80 \%$ of the cases. ${ }^{43-45}$ In the vast majority of the cases in the present study $(91.7 \%)$, surgical procedures that preserved the limbs were performed. When bone replacements were implanted, allografts were used in approximately one-fifth of the cases and unconventional endoprostheses were used in the remainder.

The surgical margins examined under the microscope were considered adequate, albeit narrow in some cases, ranging from 1 to 10 millimeters with a mean of $0.26 \mathrm{~cm}$. The margin variable was not shown to be a significant prognostic factor in the present study. Narrow margins can be explained for surgical procedures. In the majority of cases, the limbs were preserved despite the fact that some of the tumors were quite large. Importantly, we had a low rate of recurrence $(8.3 \%)$.

According to the literature, the local recurrence rate in osteosarcoma cases ranged from 2 to $10 \% .^{11,20,24,46}$ This recurrence rate was also observed for the cases treated by Brazilian surgeons, despite the large dimensions of this type of tumor at the time of diagnosis in almost all series. ${ }^{10,15}$

The five- and ten-year OS rates in GBTO studies III and IV were lower than the rates reported by American and European studies. ${ }^{15}$ However, in our study (GBTO Study V), one needs to bear in mind that the respective two- and fiveyear survival rates of $61 \%$ and $55 \%$, respectively, relate to a group of individuals with a poor response to chemotherapy and who have a tendency towards lower survival rates. Nevertheless, the estimated five-year OS rate of study V $(55 \%)$ was greater than that of the preceding two studies (III and IV: $50.1 \%$ ), thus indicating a trend towards improved survival among patients with osteosarcoma who were treated within our setting.

\section{CONCLUSIONS}

In this series, during our follow-up:

1. Tumor size greater than $15 \mathrm{~cm}$ was found to be an adverse factor for LRFS and OS but did not influence the MFS.

2. The histological type of osteosarcoma is a significant independent predictor for LRFS, MFS and OS.

\section{REFERENCES}

1. Mehlman CT, Cripe TP. Osteosarcoma. In: eMedicine-Osteosarcoma [online]. Revisado em 28/03/2008. Disponível em: http:/www. emedicine.com/orthoped/topic531.htm. Acesso em 28 março 2009.

2. Dorfman HD, Czerniak B. Bone Cancers. Cancer. 1995;75:203-10.

3. Forest M, De Pinieux G, Knuutila S. Secondary osteosarcoma. In: Fletcher CDM, Unni KK, Mertens F, editores. Pathology and genetics of tumours of the soft tissues and bones. Lyon: IARC Press; 2002. p.277-8.

4. Dahlin DC, Unni KK. Bone tumor: General aspects and data on 11,087 cases. 5th ed Philadelphia: Lippincott-Raven; 1996. Chapter 11, p.14378: Osteosarcoma.

5. Dorfman HD, Czerniak B. Bone Tumors. 1th ed. St Louis: Mosby; 1998. Chapter 5, p.128-247: Osteosarcoma.

6. Raymond AK, Ayala AG, Knuutila S. Secondary osteosarcoma. In: Fletcher CDM, Unni KK, Mertens F, editores. Pathology and genetics of tumours of the soft tissues and bones. Lyon: IARC Press; 2002. p.264-70.
7. Provisor AJ, Ettinger LJ, Nachman JB, Krailo MD, Makley JT, Yunis EJ, et al. Treatment of nonmetastatic osteosarcoma of the extremity with preoperative and postoperative chemotherapy: a report from Children's Cancer Group. J Clin Oncol. 1997;15:76-84.

8. Link MP, Gebhardt MC, Eilber F. Osteosarcoma. In: Pizzo, PA; Poplack, DG. Principles and Practice of Pediatric Oncology. Philadelphia: Lippincott Williams \& Wilkins; 4th Edition, 2002. p. 889-920.

9. Dahlin DC, Coventry MB. Osteogenic sarcoma: A study of six hundred cases. J Bone Joint Surg Am. 1967;49:101-10.

10. Petrilli AS, Gentil FC, Epelman S, Lopes LF, Bianch A, Lopes A, et al. Increased survival, limb preservation, and prognostic factors for osteosarcoma. Cancer. 1991;68:733-7.

11. Glasser DB, Lane JM, Huvos AG, Marcove RC, Rosen G. Survival, prognosis and therapeutic response in osteogenic sarcoma. The Memorial Hospital experience. Cancer. 1992;69:698-708. 
12. Fuchs N, Bielack SS, Epler D, Bieling P, Delling G, Körholz D, et al. Long-term results of the cooperative German-Austrian-Swiss osteosarcoma study group's protocol COSS-86 of intensive multidrug chemotherapy and surgery for osteosarcoma of the limbs. Ann Oncol. 1998;9:893-9.

13. Ferrari S, Bertoni F, Mercuri M, Picci P, Giacomini S, Longhi A, et al. Predictive factors of disease- free survival for nonmetastatic osteosarcoma of the extremity: an analysis of 300 patients treated at the Rizzoli Institute. Ann Oncol. 2001;12:1145-50.

14. Rech A, Castro Junior CG, Mattei J, Gregianin L, Di Leone L, David A, et al. Clinical features in osteosarcoma and prognostic implications. J Pediatr (Rio J). 2004;80:65-70.

15. Petrilli AS, de Camargo B, Filho VO, Bruniera P, Brunetto AL, JesusGarcia R, et al. Results of the Brazilian Osteosarcoma treatment group studies III and IV: prognostic factors and impact on survival. J Clin Oncol. 2006;24:1161-8.

16. Mertens F, Mandahl N, Orndal C, Baldetorp B, Bauer HC, Rydholm A, et al Cytogenetic findings in 33 osteosarcomas. Int J Cancer. 1993;55:4450 .

17. Guerra RB, Tostes MD, Miranda LC, Camargo OP, Baptista AM, Caiero MT, et al. Comparative analysis between osteosarcoma and Ewing's sarcoma: evaluation of the time from onset of signs and symptoms until diagnosis. Clinics. 2006;61:99-106.

18. Rosen G, Marcove RC, Caparros B, Nirenberg A, Kosloff C, Huvos AG. Primary osteogenic sarcoma: the rationale for preoperative chemotherapy and delayed surgery. Cancer. 1979;43:2163-77.

19. Hudson M, Jaffe MR, Jaffe N, Ayala A, Raymond K, Carrasco H, et al. Pediatric osteosarcoma: therapeutic strategies, results, and prognostic factors derived from 10-year experience. J Clin Oncol. 1990;8:1988-97.

20. Bramwell VHC, Burgers M, Sneath R, Souhami R, van Oosterom AT, Voût PA, et al. A comparison of two short intensive adjuvant chemotherapy regimens in operable osteosarcoma of limbs in children and young adults: The first study of the European Osteosarcoma Intergroup. J Clin Oncol. 1992;10:1579-91.

21. Meyers PA, Heller G, Healy J, Huvos A, Lane J, Marcove R, et al. Chemotherapy for nonmetastatic osteogenic sarcoma: The Memorial Sloan-Kettering experience. J Clin Oncol. 1992;10:5-15.

22. Bacci G, Picci P, Ferrari S, Ruggieri P, Casadei R, Tiengui A, et al. Primary chemotherapy and delayed surgery for non metastatic osteosarcoma of the extremity. Results in 164 patients preoperatively treated with high doses of methotrexate, followed by cisplatin and doxorubicin. Cancer. 1993;72:3227-38.

23. Souhami RL, Craft AW, Van der Eijken JW, Nooij M, Spooner D, Bramwell VH, et al. Randomized trial of two regimens of chemotherapy in operable osteosarcoma: A study of the European Osteosarcoma Intergroup. Lancet. 1997;350:911-7.

24. Bacci G, Ferrari S, Bertoni F, Ruggieri P, Picci P, Longhi A, et al. Long-term outcome for patients with nonmetastatic osteosarcoma of the extremity treated at Istituto Ortopedico Rizzoli according to the Instituto Ortopedico Rizzoli/osteosarcoma-2 protocol: an updated report. J Clin Oncol. 2000;18:4016-27.

25. Bielack S, Kempf-Bielack B, Delling G, Exner GU, Flege S, Helmke K, et al. Prognostic factors in high-grade osteosarcoma of the extremities or trunk: An analysis of 1702 patients treated on neoadjuvant cooperative osteosarcoma study group protocols. J Clin Oncol. 2002;20:776-90.
26. Petrilli AS, Penna V, Lopes A et al. IIIB osteossarcoma. Current management, local control, and survival statistics. Clin Orthop. 1991;270:60-6.

27. Baptista AM, Camargo OP, Croci AT, Oliveira CRGCM, Azevedo Neto RS, Gianotti MA, et al. Synovial sarcoma of the extremities: prognostic factors for 20 nonmatastatic cases and a new histologic grading system with prognostic significance. Clinics. 2006;61:381-6.

28. Bispo Júnior RZ, Camargo OP, Oliveira CRGCM, Filippi RZ, Baptista AM, Caiero MT. Prognostic factors and expression of MDM2 in patients with primary extremity liposarcoma. Clinics. 2008;63:157-64.

29. Huvos AG, Rosen G, Marcove RC. Primary osteogenic sarcoma: pathologic aspects in 20 patients after treatment with chemotherapy en bloc resection, and prosthetic bone replacement. Arch Pathol Lab Med. 1977;101:14-8.

30. Enneking WF, Spanier SS, Goodman MA. Current concepts review. The surgical satging of musculoskeletal sarcoma. J Bone Joint Surg Am. 1980;62:1027-30.

31. Davis AM, Bell RS, Goodwin PJ. Prognostic factors in osteosarcoma: a critical review. J Clin Oncol. 1994;12:423-31.

32. Pakos EE, Nearchou AD, Grimer RJ, Koumoullis HD, Abudu A, Bramer JAM, et al. Prognostic factors and outcomes for osteosarcoma: an international collaboration. Eur J Cancer. 2009, doi:10.1016/j. ejca.2009.03.005

33. Raymond AK, Chawla SP, Carrasco CH, Ayala AG, Fanning CV, Grice B, et al. Osteosarcoma chemotherapy effect: A prognostic factor. Semin Diagn Pathol. 1987;4:212-36.

34. Spanier SS, Shuster JJ, Vander Griend RA. The effect of local extent of the tumor on prognosis in osteosarcoma. J Bone Joint Surg Am. 1990;72:643-53.

35. Goorin AM, Perez-Atayde A, Gebhardt M, Andersen JW, Wilkinson $\mathrm{RH}$, Delorey MJ, et al. Weekly high-dose methotrexate and doxorubicin for osteosarcoma: The Dana-Farber Cancer Institute/The Children's Hospital-Study III. J Clin Oncol. 1987;5:1178-84.

36. Bieling P, Rehan N, Winkler P, Helmke K, Maas R, Fucs N, et al. Tumor size and prognosis in aggressively treated osteosarcoma. J Clin Oncol. 1996;14:848-58.

37. Simon R. Clinical prognostic factors in osteosarcoma. Cancer Treat Rep. 1978;62:193-7.

38. Bacci G, Ferrari S, Longhi A, Forni C, Zavatta M, Versari M, et al. High grade osteosarcoma of the extremity: Differences between localized and metastatic tumors at presentation. J Pediatr Hematol Oncol. 2002;24:2730 .

39. Saeter G, Elomaa I, Wahlqvist Y, Alvegard TA, Wiebe T, Monge O, et al. Prognostic factors in bone sarcomas. Acta Orthop Scand Suppl. 1997;273:156-60

40. Hauben EI, Weeden S, Pringle J, Van Marck EA, Hogendoorn PC. Does the histological subtype of high-grade central osteosarcoma influence the response to treatment with chemotherapy and does it affect overall survival? A study on 570 patients of two consecutive trials of the European Osteosarcoma Intergroup. Eur J Cancer. 2002;38:1218-25.

41. Bacci G, Bertoni F, Longhi A, Ferrari S, Forni C, Biagini R, et al. Neoadjuvant chemotherapy for high-grade central osteosarcoma of the extremity histologic response to preoperative chemotherapy correlates with histologic subtype of the tumor. Cancer. 2003;97:3068-75. 
42. Wuisman P, Enneking WF, Roesner A. Local growth and the prognosis of osteosarcoma. Int Orthop. 1992;16:55-8.

43. Link MP, Goorin AM, Horowitz M, Meyer WH, Belasco J, Baker A, et al. Adjuvant chemotherapy of high grade osteosarcoma of the extremity. Updated results of the Multiinstitutional Osteosarcoma Study. Clin Orthop Relat Res. 1991;270:8-14.

44. Sim FH, Frassica FJ. General perspectives, pathogenesis, pathophysiology and skeletal disfuncion. Orthopaedics. 1992;15:599-604.
45. Bacci G, Ferrari S, Longhi A, Perin S, Forni C, Fabbri N, et al. Pattern of relapse in patients with osteosarcoma of the extremities treated with neoadjuvant chemotherapy. Eur J Cancer. 2001;37:23-8.

46. Picci P, Capanna R, Bacci G, Mercuri M, Biagini R, Ruggieri P, et al. Margins, necrosis and local recurrence after conservative surgery in osteosarcoma. Chir Organi Mov. 1990;75(1 Suppl):82-5. 